REFERENCES:

[1] Dubois EL, Tuffanelli DL. Clinical Manifestations of Systemic Lupus Erythematosus. Computer Analysis of 520 Cases. JAMA 1964; 190:112-19

[2] Cervera R. Khamashta M, Font J, et al. Systemic Lupus Erytematosus: Clinical and Immunologic Patterns of Disease Expression in a Cohort of 1,000 Patients. Medicine 1993; 72(2):113-24

[3] Shapira Y, Weinberger A, Wysenbeek AJ. Lymphadenopathy in Systemic Lupus Eritematosus. Prevalence and Relation to Disease Manifestations. Clin Rheumatol 1996; 15(4):335-38

Disclosure of Interest: None declared

DOI: 10.1136/annrheumdis-2018-eular.4125

\section{AB0548 ANTIBODY TO PURINNUCLEOSOID PHOSPHILILASE CAN BE USED AS AN ADDITIONAL MARKER OF INFECTIOUS COMPLICATIONS WITH SYSTEMIC LUPUS ERYTHROMATOSIS}

A.V. Aleksandrov, E.V. Benedickaya, I.Y. Alekhina, N.V. Aleksandrova. Research Institute of Clinical and Experimental Rheumatology named after A.B. Zborovsky, Volgograd, Russian Federation

Background: Purine nucleoside phosphorylase (PNP, EC 2.4.2.1) plays a leading role in the assimilation of nucleosides and nucleotides by the cell, as well as in maintaining the immune status of the organism. Patients with PNP deficiency are highly susceptible to various infections, in view of the fact that the decreased activity of PNP is closely related to the insufficiency of cellular immunity.

Objectives: Study of the possibility of using the level antibodies to purine nucleoside phosphorylase as an additional marker of infectious complications in patients with systemic lupus erythematosus (SLE).

Methods: The study included 60 patients with SLE (women - 91.7\%, mean age $36.32 \pm 15.27$ years, average duration of the disease $7.96 \pm 7.35$ years) with different clinical manifestations (SLEDAl activity 8.93 $\pm 5,74$, ECLAM activity $5.30 \pm 2.79$, damage index SLICC/ACR 1.95 \pm 1.71 ). Antibodies to PNP (anti-PNP) were determined in our indirect ELISA test using the immobilised form of the enzyme as an antigenic matrix.

The presence of infectious complications in SLE patients was assessed by characteristic clinical manifestations and was considered to be confirmed when the causative agent was recognised and/or the serological analyses are positive. Infections were diagnosed in $38.3 \%$ of patients, the most frequent localization was the urinary system $(n=12 ; 52.2 \%)$ and female genitalia $(n=5 ; 21.7 \%)$

Results: In the presence anti-PNP in the blood serum of patients with SLE we have often noted infectious complications $\left(p=0.025\right.$, criterion $\left.\chi^{2}\right)$ and less often lung damage $(p=0.17)$ (when compared with a group of patients with SLE seronegative for the presence of anti-PNP).

Statistically significant differences (Mann-Whitney U-criterion) were detected only for pyuria $(p<0.0005), C$-reactive protein level $(p=0.048)$ and the number of ceruloplasmin ( $p=0.008$ ), while comparing the groups of SLE patients with the presence of infectious complications and without it; differences in the level of antibodies to CP $(p=0.052)$ did not reach statistical significance. Other clinical and laboratory parameters do not have statistically significant differences between the two sessions. This fact complicates the recognition and differential diagnosis of infectious complications in SLE significantly.

The analysis of the characteristic curve of antibodies to PNP in the diagnosis of infectious pathology in SLE shows limited possibilities for using this parameter. The square under the curve was 0.687 (95\% confidence interval $0.523-0.804$, $\mathrm{p}=0.008$ ), the separation point was $0.386 \mathrm{U} / \mathrm{ml}$ with a sensitivity of $55 \%$ and a specificity of $83 \%$.

Taking into account that the typical clinical manifestations of infection with SLE can be erased as a result of the actual activity of SLE, and due to the use of immunosuppressive drugs, timely diagnosis and treatment become especially relevant. This study indicates the possibility of using the level of antibodies to PNP as an additional marker of infection, but the balance between specificity and sensitivity of this test does not allow to recommend it as a single study.

Conclusions: A high level of anti-PNP in the serum, especially combined with the C-reactive protein increase and in the absence of other markers of SLE activation allows to suspect the presence of infection and points to the need for in-depth research directed to identify the pathogen.

Disclosure of Interest: None declared

DOI: 10.1136/annrheumdis-2018-eular.5276

\section{$\mathrm{AB} 0549$}

CAN THE OVERALL THROMBOTIC RISK IN SYSTEMIC LUPUS ERYTHEMATOSUS BE DETERMINED? THE COMBINED ROLE OF CLASSIC CARDIOVASCULAR FACTORS AND ANTIPHOSPHOLIPID ANTIBODIES

Â. S. Mota, I. Cunha, J. Andrade, C. Eira, S. Machado, J. Tavares, V. Romão, R. Silvério, C. Martins. Medicina, CENTRO HOSPITALAR TONDELA-VISEU, E.P. E., Viseu, Portugal

Background: Systemic Lupus Erythematosus (SLE) is a chronic inflammatory disorder. Antiphospholipid syndrome (APS) is a thrombotic disorder associated with the presence of antiphospholipid antibodies (APA) and increased cardiovascular risk (CVR). Framingham (FRM) and SCORE (Systematic Coronary Risk Evaluation) scales are available CVR assessment systems. aGAPSS (adjusted Global AntiphosPholipid Syndrome Score) combine positive APA and CVR factors, which was suggested to determine the thrombotic risk in persistently positive APA (PPAPA) patients.

Objectives: To determine the role of the thrombotic factors in SLE patients, considering CVR factors and APA. To access the application of different thrombotic risk scales.

Methods: A retrospective cohort study of 84 patients with SLE followed in an outpatient setting of a Portuguese central hospital was performed. The study evaluated patient gender, current age, age at diagnosis, duration of illness, presence of another autoimmune disease (AID), CVR factors [obesity (OB), diabetes (DB), arterial hypertension (AH), dyslipidaemia (DL), smoking (SM)], presence of APA, treatment, dose and duration of steroids. The FRM, SCORE and aGAPSS scores were calculated. The data was analysed using SPSS and considered significant if $\mathrm{p}<0.05$.

Results: Table 1 characterises the study population. Male patients had a highe prevalence of $\mathrm{AH}(\mathrm{p}=0.022), \mathrm{DL}(\mathrm{p}=0.047)$, and $\mathrm{SM}(\mathrm{p}=0.001)$, with a risk of $11 \%$ $20 \%$ in the FRM scale and a risk of $5 \%-14 \%$ in the SCORE $(p=0.000)$. Female patients had a higher presence of another AID $(p=0.014)$ and treatment with disease-modifying antirheumatic drugs $(p=0.014)$. FRM scale reveals a risk of $11 \%-$ $20 \%$ in the presence of $\mathrm{AH}$ and $>20 \%$ in SM $(p=0.001)$. The SCORE scale reveals a risk of $5 \%-9 \%$ in the presence of $\mathrm{AH}(\mathrm{p}=0.003)$ and $10 \%-14 \%$ in $\mathrm{DL}(\mathrm{p}=0.024)$. When the risk is $6 \%-20 \%$ in the FRM scale, the risk is lower in SCORE $(p=0.000)$ APA does not correlate with an increased CVR. All APA are associated with another AID, APS and PPAPA. The aGAPSS associates a score of 7-12 if another AID is present $(p=0.000)$; 4-9 with APS; $>7$ with PPAPA $(p=0.000) ; 4-6$ with $\mathrm{DB}(\mathrm{p}=0.039), \mathrm{DL}(\mathrm{p}=0.002)$ and $\mathrm{AH}(\mathrm{p}=0.000) ;>7$ with lupus anticoagulant $(\mathrm{LA}),>7$ with anticardiolipin antibodies $(\mathrm{aCL})$ and $>12$ with anti- $\beta-2$ glycoprotein-I antibody (antiß2GPI) $(p=0.000)$.

Abstract AB0549 - Table 1

\begin{tabular}{lc}
\hline Women & $74 \%$ \\
\hline Current age & $52.3 \pm 16.2$ years \\
Age of diagnosis & $44.9 \pm 17.4$ years \\
Duration of disease & $7.2 \pm 7.5$ years \\
Another AID & $12 \%$ \\
PPAPA & $30 \%$ (APS $60 \%$, Sjögren syndrome 36\%) \\
CVR & AH 42.8\%, DL $21.4 \%$, OB $11.9 \%$, DB $10.7 \%$, SM $9.5 \%$ \\
APA & LA 19\%, aCL $15.5 \%$, antiß2GPI $7.1 \%$ \\
Steroid use & $61 \%$ \\
DMARDs & $89 \%$ \\
\hline
\end{tabular}

Conclusions: This study highlights the existence of thrombotic factors in SLE. Their risk is even more elevated when another AID is present. The FRM and SCORE scales reflects the CVR. In SLE patients both the CVR factors and the presence of APA must be evaluated. Therefore, not only should the FRM scale be calculated, but also the global thrombotic risk, using the aGAPSS, must be accessed.

\section{REFERENCES}

[1] Sarzi-Puttini P, Atzeni F, Carrabba M. Cardiovascular risk factors in systemic lupus erythematosus and in antiphosphlipid syndrome. Minerva Med 2003;94(2):63-70.

[2] Sciascia S, Radin M, Sanna G, Cecchi I, Roccatello D, Bertolaccini ML. Clinical utility of the global anti-phospholipid syndrome score for risk stratification: a pooled analysis. Rheumatology 2018.

Disclosure of Interest: None declared

DOI: 10.1136/annrheumdis-2018-eular.7389 


\section{AB0550 CLINICAL-EPIDEMIOLOGICAL FEATURES OF PATIENTS WITH A LATE-ONSET LUPUS IN A TERTIARY CARE HOSPITAL}

A. Crespo Golmar, C. Moriano, M. Garijo, C. Iñiguez, I. González, A. López, E. Díez, C. Álvarez, M. Martín, E. Vallejo, T. Pérez. Rheumatology Department, University Health Care Complex of León, León, Spain

Background: Different frequency of clinical and serological manifestations has been detected according to the age of onset of the patients with Systemic lupus erythematosus (SLE). According to the literature, senile SLE manifests between $6 \%$ and $18 \%$ of the patients with lupus.

Objectives:

1. To identify and analyse the clinical-serological and epidemiological features of senile SLE in our environment.

2. To determine the average survival time and mortality in these patients, identifying its main cause.

Methods: Observational retrospective study of 319 patients diagnosed with SLE (according to ACR 1992 and SLICC 2012 criteria) at the Hospital of León between 1997-2017 and with an age of onset $\geq 65$ years, obtaining a total of 68 patients with senile SLE.

Results: The mean age at diagnosis was $75.4 \pm 12.1$ years, with a female/male ratio of 2.4. The most frequent manifestations were as joint (63.2\%) and haematological manifestations in the form of leuco-lymphopenia (55.9\%). The hemolytic anaemia only appeared in $2.9 \%$ of the cases and the thrombocytopenia in $25 \%$. $36.8 \%$ of patients showed photosensitivity and $29 \%$ had other cutaneous manifestations, being the malar erythema the most prevalent type (60\%), followed by the discoid lupus erythematosus $(20 \%)$ and the subacute lupus (15\%). Alopecia was only observed in $4.4 \%$. Lupus nephritis was detected in the form of proteinuria in $4.4 \%$ of the patients, and only one patient had microscopic haematuria. Lung involvement was uncommon (8.8\%), taking precedence the UIP (33.3\%) over the rest of the pulmonary manifestations. Only $11.1 \%$ of the patients with senile SLE had serositis, being in the form of pleuritis in $75 \%$ of the cases, pericarditis in the $37.5 \%$ and ascites in the $12.5 \%$. Regarding the neurological involvement, 5 patients showed polyneuropathy and 1 had chorea. Likewise, the frequency of Sjögren, Raynaud and secondary antiphospholipid syndrome was of $16.7 \%$, respectively.

The most important serological findings were: $97.3 \%$ ANA; $44.1 \%$ DNA and $20.6 \%$ hypocomplementemia, with $54.4 \%$ of the patients having serological activity. Only $5.9 \%$ had anti-Sm. Antiphospholipid antibodies were positive in $41.2 \%$ of the cases, with $4.4 \%$ of them showing triple positivity.

The average survival time was of 13.7 years (SD: 10.9-16.5). Out of the total patients, 14 died (20.59\%), mostly due to infectious etiology (35.7\%) and $14.28 \%$ due to disease activity. Other less common causes were neoplasia or ischaemic heart disease (7.14\% respectively)

Conclusions:

- The late-onset SLE prevails in our environment, one of every 5 patients diagnosed with SLE in our consulting room is older than 65 years.

- It is found most often in women and it is confirmed a lower male/female ratio than expected.

- Joint and haematology manifestations and cutaneous involvement in the form of malar erythema define the clinical profile of our patients with senile SLE, with the renal involvement or the presence of serositis being uncommon.

- Half of the patients had serological activity at the onset, having hypocomplementemia only in 1 out of 5 cases.

- Infections were the first cause of mortality in our sample with an average survival time of around 13 years.

Disclosure of Interest: None declared

DOI: 10.1136/annrheumdis-2018-eular.7538

\section{AB0551 OBSTETRICAL MORBIDITY RELATED TO ANTI-SSA ANTIBODIES: DATA FROM A FRENCH MONOCENTRIC RETROSPECTIVE STUDY}

L. Plaçais ${ }^{1}$, F. Carrat ${ }^{2}$, L. Carbillon ${ }^{3}$, P. Nicaise ${ }^{4}$, A. Benbara ${ }^{5}$, O. Fain ${ }^{1}$, A. Mekinian ${ }^{1}{ }^{1}$ Internal Medicine; ${ }^{2}$ Epidemiology and Public health, Saint Antoine hospital; ${ }^{3}$ Obstetrics and Gynecology, Jean Verdier hospital; ${ }^{4}$ Auto-immunity and hypersensibility, Bichat hospital, ${ }^{5}$ Obstetrics and Gynaecology, Jean Verdier hospital, Paris, France

Background: Obstetrical morbidity is an issue among autoimmune diseases patients. Anti-SSA positivity is well known for it's effect on fetus and risk of congenital heart block but no evidence has been yet found for its effect on obstetrical morbidity

Objectives: To investigate anti-SSA positivity effect among a large monocentric cohort of obstetrical morbidity patients and to evaluate the efficacity of different treatment regimens

Methods: All women who were seen from January 2010 to January 2015 in JeanVerdier University Hospital for obstetrical morbidities were retrospectively included. All patients had been tested for anti-nuclear antibodies and anti-SSA/Ro and anti-SSB/La antibodies.

Results: We included 244 women (median age 34 years [interquartile range $21-$ 53]) with 869 pregnancies overall. In 27 (11\%) patients with anti-SSA antibodies, the median age at the time of pregnancy was $29.5(17-40)$ years, with mean number of 3.66 pregnancies per woman. For these 27 patients, 83/99 pregnancies (84\%) had an adverse obstetrical outcome: fetal loss ( $n=65$; at a median of 20 (438) weeks' gestation), preeclampsia $(n=15)$, intrauterine growth restriction $(n=7)$, prematurity $(n=7)$, and congenital heart block $(n=2)$. In comparing women with obstetrical antiphospholipid syndrome and unexplained adverse complications, there was no major difference in frequency of recurrent miscarriages, preterm deliveries, preeclampsia or fetal loss. Among factors associated with obstetrical outcome and fetal loss, aspirin and hydroxychloroquine treatments were significantly associated with a favourable obstetrical outcome: odds ratio $0.05[95 \%$ confidence interval $0.01 ; 0.37](\mathrm{p}=0.003)$ and $0.15[0.02 ; 0.98](\mathrm{p}=0.04)$.

Conclusions: Women with unexplained recurrent obstetrical complications should be screened for anti-SSA antibodies. The benefit of aspirin and hydroxychloroquine treatment to improve the obstetrical outcome should be confirmed in prospective studies.

Disclosure of Interest: None declared

DOI: 10.1136/annrheumdis-2018-eular.5580

\section{AB0552 THE CLINICAL AND LABORATORY FEATURES OF ANTIPHOSPHOLIPID ANTIBODY POSITIVE PATIENTS WITH OR WITHOUT SYSTEMIC LUPUS ERYTHEMATOSUS}

A. Yazici ${ }^{1}$, A. Omma ${ }^{2}$, S. Yilmaz ${ }^{3}$, S. Yavuz ${ }^{4}$, K. Ayar ${ }^{5}$, E. Kasapoglu ${ }^{6}$, B. Kisacik ${ }^{7}$, A. Cefle ${ }^{1}$, D. Erkan ${ }^{8} .{ }^{1}$ Rheumatology, Kocaeli University School of Medicine, Kocaeli; ${ }^{2}$ Rheumatology, Numune Hospital; ${ }^{3}$ Rheumatology, Gulhane Training and Research Hospital, Ankara; ${ }^{4}$ Rheumatology, Bilim University School of Medicine, Istanbul; ${ }^{5}$ Rheumatology, University of Health Sciences Yüksek ihtisas Training and Research Hospital, Bursa; ${ }^{6}$ Rheumatology, Göztepe Training and Research Hospital, Istanbul; ${ }^{7}$ Rheumatology, Medicalpark Hospital, Gaziantep, Turkey; ${ }^{8}$ Barbara Volcker Center for Women and Rhumatic Diseases, Hospital for Special Surgery, Weill Cornell Medicine, New York, USA

Background: A multicenter antiphospholipid antibody (aPL) clinical database was created in 2016 with the pariticipation of six rheumatology centres around the country. The purpose has been to better define aPL-related clinical manifestations and management strategies; and also to establish a resource for future clinical studies.

Objectives: In this first analysis, we compared the clinical and laboratory features of aPL-positive patients with and without systemic lupus erythematosus (SLE). Methods: The demographic, clinical, laboratory, treatment characteristics of the aPL-positive patients with/without other systemic autoimmune diseases (SAID) are recorded at enrollment according to a predefined protocol. The inclusion criteria are positive aPL (lupus anticoagulant test [LA], anticardiolipin antibody [aCL], and/or anti $\beta_{2}$-glycoprotein-I antibody [a $\left.\beta_{2} \mathrm{GPI}\right]$ ) based on the Updated Sapporo Antiphospholipid Syndrome (APS) Classification Criteria at least twice within one year prior to enrolment. ${ }^{1,2}$ For the purpose of this analysis, we only included aPLpositive patients without other autoimmune diseases (primary aPL/APS) and aPLpositive SLE patients (SLE aPL/APS).

Results: As of January 2018, 105 aPL-positive patients were recruited (mean age: $42.6 \pm 10.1$ [min-max: 19-70]; 83 [79\%] female; and 67 [64\%] with another SAID). Ten patients were excluded from the analysis due to their SAID history other than SLE. Of the remaining 95 patients, $38(40 \%)$ had primary aPL/APS; 57 (60\%) fulfilled the ACR SLE Classification Criteria; 42 (44\%) had thrombotic APS (TAPS) (8 arterial, 24 venous, and 8 both); 21 (22\%) had obstetric APS (OAPS) $22(23 \%)$ had both TAPS and OAPS (7 arterial, 14 venous, and 1 both); and 10 (11\%) had no TAPS/OAPS. Fifty percent of the patients had history of at least one non-criteria aPL-manifestation. Demographics, clinical and laboratory manifestations, and medications were similar between primary aPL/APS and SLE aPL/APS patients except increased frequency of autoimmune hemolytic anaemia, aCL IgG, and hydroxychloroquine use in SLE aPL/APS patients (table 1). 